\title{
Editorial corner - a personal view \\ Science Babel: Does the lack of a common terminology in the field of emulsion templating hinder progress?
}

\author{
K. Kim, A. Bismarck* \\ Department of Chemical Engineering, Polymer and Composite Engineering (PaCE) Group, Imperial College London, \\ South Kensington Campus, London, SW2 7AZ, UK
}

Emulsion templating to produce macroporous polymers with a well defined structure has developed into an established research area. Potential applications of very concentrated emulsions and macroporous polymers made from them rapidly expanded from food preparation to cosmetics and from oil recovery and to tissue engineering, respectively. In particular, high internal phase emulsions (HIPEs) are widely used as template to create very high porosity macroporous polymers, commonly known as poly(merised)HIPEs. Emulsion templating has proved to be extremely useful to manufacture tailor made highly interconnected open porous polymers. The advantage of this method is that the template is liquid which allows moulding it to any desired shapes. Polymer foams produced by emulsion templating have been explored for many applications. For instance, polyHIPEs based on biodegradable components show excellent biocompatibility for a wide range of cell types.

As the field is growing, many researchers have joined and produced remarkable research outcomes. More and more research groups are producing new templates and polymer foams for numerous applications but there are growing concerns about the non-consensual use of terminology. The very nature of making new materials inspires researchers to create new names to refer to their research outcomes on the basis of potential applications. This has become reality now: Researchers use different terminology for the emulsion templates, ranging from concentrated emulsions to HIPEs. Even if polymerised the resulting polymers were given different names, such as polyHIPE, macroporous polymers and polyFoams. Researchers also refer to an identical pore structure of 'polyHIPEs' with different names. There are plenty of examples causing potential confusion; for instance, the 'spherical' cavities in the macroporous polymers are called differently: pores, voids, cells and cavities. Moreover, the 'holes' in the polymer walls forming at contact points of neighbouring droplets during the polymerisation are called by some researchers pore throats, another research team names them windows. However, recently it becomes more complicated as researchers call them interconnecting pores or intercellular pores.

At the current stage, researchers seem not to be bothered by this diversified usage of terminology. However, as the field is growing, the confusion could cause babelisation of the field. As researchers produce interesting and promising outcomes, they are enticed to baptise their own creations. Before reaching the incommensurable state between researchers, the field needs to be unified with a well defined terminology for referring to new materials. The game of name is a part of progressing science but it might hinder progress in this interdisciplinary field as it becomes harder to identify what others have done - leading potentially to reinvent the wheel. 